\title{
Structural, rheological and dynamics insights of hydroxypropyl guar gel-like systems
}

\author{
Chiara Berlangieri ${ }^{\mathrm{a}}$, Giovanna Poggi ${ }^{\mathrm{a}}$, Sergio Murgia ${ }^{\mathrm{b}}$, Maura Monduzzi ${ }^{\mathrm{b}}$, Luigi Dei ${ }^{\mathrm{a}}$, \\ Emiliano Carretti ${ }^{\mathrm{a}, *}$ \\ a Department of Chemistry “Ugo Schiff” E CSGI Consortium, University of Florence, via della Lastruccia, 3, 50019 Sesto Fiorentino, Florence, Italy \\ ${ }^{\mathrm{b}}$ Department of Chemical and Geological Sciences and CSGI, University of Cagliari, ss 554 bivio Sestu, 09042 Monserrato, Cagliari, Italy
}

\section{A R T I C L E I N F O}

\section{Article history:}

Received 3 October 2017

Received in revised form 9 January 2018

Accepted 11 February 2018

Available online 13 February 2018

\section{Keywords:}

Guar gum

Borax

Hydrogel

Cleaning

Cultural heritage conservation

\begin{abstract}
A B S T R A C T
A dynamic, rheological, and structural characterization of aqueous gel-like systems containing hydroxypropyl guar gum (HPG), borax and glycerol is presented in this paper. The role of glycerol, which is introduced as a plasticizer in the formulation, is investigated by means of ${ }^{11} \mathrm{~B}$ NMR and ${ }^{1} \mathrm{H}$ NMR PGSTE measurements in order to clarify its contribution to the gel network formation and its interaction with borax, with whom it forms a complex. The effect of gels components on the rheological behaviour and on the activation energy related to the relaxation process of the system was assessed by means of rheology. The results obtained suggest that the mechanical properties of these gels can be tuned and controlled by modulating the formulation in a wide range of compositions. Moreover, a structural characterisation has been also carried out by means of Small Angle X-ray Scattering (SAXS) to highlight the role of the various components on the mesh size of the network. The structural and mechanical characteristics of these systems suggest their potential use for applicative purposes. In this regard, one of the gel set up has been successfully tested as cleaning agent on the surface of a XIX stucco fragment coming from the La Fenice theatre (Venice, Italy) for the removal of a dirt layer composed by dust and particulated matter originated during a fire in 1996.
\end{abstract}

(C) 2018 Elsevier B.V. All rights reserved.

\section{Introduction}

Hydroxypropyl guar gum (HPG) is a derivative of guar gum in which several free hydroxyls are substituted by hydroxypropyl groups upon etherification (Fig. 1A). It is a natural polysaccharide extracted from the seeds of Cyamopsis tetragolonoba, and it is composed by a linear chain of mannose units linked by $\beta-1,4$ bonds. This backbone is functionalized by galactose residues $\alpha-1,6$-linked, randomly attached, forming short side-branches. It is cheap, nontoxic, biocompatible, biodegradable, easy available and soluble in water, where it forms viscous solutions even at low concentrations [1-5]. For these reasons guar gum has several applications in food, paper, textile, pharmaceutical and petroleum industries.

HPG together with similar polysaccharides has been recently used for the formulation of versatile and well-performing materials with various fields of application [1,6-10]. Gel-like systems containing ionic surfactants and modified guar covalently crosslinked

\footnotetext{
* Corresponding author.

E-mail addresses: carretti@csgi.unifi.it, emiliano.carretti@unifi.it (E. Carretti).
}

have also been proposed as agents for removing solid particles, able to perform a gentle mechanical surface cleaning of sensitive materials such as ceramic, wood, glass and metals [11]. Hydrogels made by guar and HPG crosslinked with ions such as titanate and borax are widely used in the oil and gas industry, mainly as a proppant transport agent in hydraulic fracturing process [1,6-10].

Furthermore, the efficacy of borax as crosslinker for polymers bearing hydroxyl groups is well known (see Fig. 1B) [12,13]. The nature of this linkage is debated: according to the model proposed by Shibayama for polyvinyl alcohol [14] and for scleroglucans [15,16], mixed physical/chemical linkages occur; nevertheless, more recent studies suggest the presence of chemical crosslinks only between borax and polymeric chains with the formation of chemical bridges by transient covalent bonds [17,18]. Moreover, being the lifetime of these covalent crosslinks in the order of seconds [13,19], the guar-borate networks are usually characterized by self-healing properties.

It is well known that glycerol increases the flexibility of polymeric materials by increasing the interchain spacing; due to this and to its large availability, glycerol is used as plasticizer for polymeric materials in many fields, as food packaging $[20,21]$ or to 


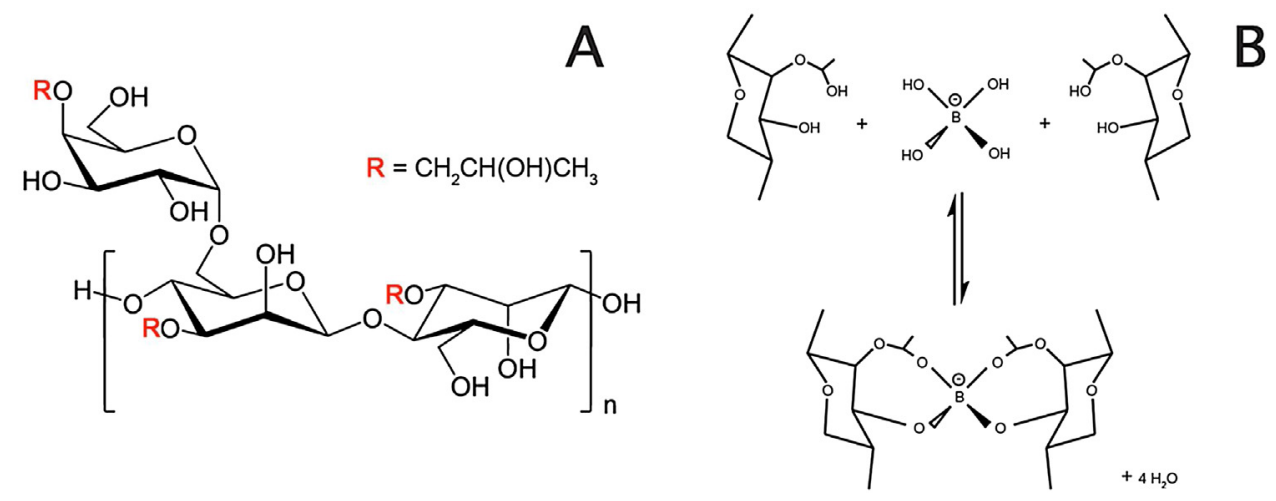

Fig. 1. (A) Chemical structure of hydroxypropyl guar (HPG). (B) Crosslinking reaction leading to the formation of a gelator network.

improve the plasticity of protein and polysaccharides based films $[20,21]$. It can also lead to an increase of the elongation at break of materials, resulting in systems that can resist to a certain stretching before breaking [20,21].

In this paper, a dynamic, rheological, and structural characterization of aqueous gel-like systems containing HPG, borax and glycerol is presented. In the last decades, several papers focusing on the study of the mechanical properties and the applicative performances of HPG aqueous solutions [22] and gel-like systems $[8-10,23,24]$ have been published. However, to our knowledge, a systematic study of the simultaneous role of both borax and glycerol on the structural and on the mechanical properties of aqueous HPG based gels, which is the main subject of this paper, is still lacking. The role of glycerol in the crosslinking mechanism and its interactions with borax were studied here systematically for the first time by using a combined approach based on both ${ }^{11} \mathrm{~B}$ NMR, ${ }^{1} \mathrm{H}$ NMR PGSTE and rheological measurements. In particular rheological investigations allowed to obtain information on the effect of gels components on the viscoelastic behaviour, on the activation energy related to the relaxation process of the system and on the critical polymeric concentration, above which an extended threedimensional polymer network is formed. Furthermore, information about the role of all the components on the structure of HPG-based systems, were obtained by a Small Angle X-ray Scattering (SAXS) investigation.

This approach allowed to identify several of the key factors that control the nature of the materials and the results obtained suggest that the mechanical properties of these gel-like fluids can be tuned by modulating their composition. The extended structural and mechanical characterization of these gel-like systems shows that their properties can be widely controlled and tuned, by tuning the concentration of the components. This feature has important applicative implications mainly in the field of Cultural Heritage conservation where the HPG/glycerol/borax systems can be used for the selective and gentle cleaning of painted surfaces of historical and artistic interest. In this regard, a preliminary applicative test was carried out on a sample collected from a stucco decoration of La Fenice theatre, in Venice. A gel-like formulation was successfully used for the removal of a dark patina resulted from the fire that almost destroyed the theatre in 1996.

\section{Materials and methods}

\subsection{Chemicals}

Hydroxypropyl guar gum (HPG, Mw $=90 \mathrm{KDa}$ ) with a substitution level of 1.2 was kindly provided by Ametech Srl; glycerol (Merck, purity 99\%) and sodium tetraborate decahydrate, (Sigma Aldrich, purity $99.5-100 \%$ ) were used as received by the suppliers.

\subsection{Samples preparation}

Samples without plasticizer were prepared through the following procedure: HPG was placed in water and then stirred and heated at $65^{\circ} \mathrm{C}$ to favour solubilization. An aqueous solution of borax at $4 \%$ was then added and the sample was stirred with a Vortex to obtain a homogenous gelation. For samples containing the plasticizer, HPG was added to a glycerol aqueous solution. In all the samples containing borax, the ratio polymer/borax was fixed to $4 / 1$ as reported in literature for the formulation of crosslinked systems $[10,23]$, except for the series in which the crosslinker effect was investigated.

\subsection{Rheological tests}

Oscillatory shear measurements were carried out on a TA Instrument Hybrid Rheometer DISCOVERY HR-3, using a plate-plate geometry (Flat Plate $40 \mathrm{~mm}$ diameter) and a Peltier for temperature control. The minimum gap between the plates at zero radial position was $500 \mu \mathrm{m}$. The cell was closed by lowering the head to the measuring position in the $\mathrm{z}$ axis force controlled mode; the maximum squeezing force was $1.0 \mathrm{~N}$. Before starting the experiments, the samples were left to equilibrate for $30 \mathrm{~min}$ at $25^{\circ} \mathrm{C}$. Frequency sweep measurements were carried out within the linear viscoelastic range ( $5 \%$ strain), determined by means of an amplitude sweep test (see Fig. S1). The storage and loss moduli (G' and G") were measured over the frequency range of $0.001-100 \mathrm{~Hz}$.

Time Temperature Superposition (TTS) procedure was carried out to observe the temperature-dependence of the mechanical properties of the viscoelastic materials. In these experiments, the Storage modulus (G') and Loss modulus (G") were monitored as a function of frequency in the linear viscoelastic region at various temperatures by means of isothermal frequency sweep tests. TTS principle implies that curves obtained at various temperatures can be superimposed by shifting the data in the vertical and/or horizontal directions; all the curves can be combined in a single master curve choosing a reference temperature $\left(T_{0}\right)$. All the systems were analysed by keeping $25^{\circ} \mathrm{C}$ as the reference temperature. The shape of the master curve does not change with temperature, but the curve appears to be only shifted $[23,25]$. The master curve generated by the software (TRIOS Data Analysis, TA Instrument) represents the time response of the material at the reference temperature. The software can analyse the shift factors using the Arrhenius Eq. (1) to calculate the Activation Energy of the relaxation process:

$\ln a_{T}=\left(-\frac{E}{R}\right)\left(\frac{1}{T}-\frac{1}{T_{0}}\right)$ 
where $\mathrm{a}_{\mathrm{T}}$ is the shift factor, $\mathrm{E}$ is the Activation Energy associated to the relaxation process, $\mathrm{R}$ is the gas constant, $\mathrm{T}$ is the measurement temperature and $\mathrm{T}_{0}$ is the reference temperature.

\subsection{NMR measurements}

${ }^{1} \mathrm{H}$ and ${ }^{11} \mathrm{~B}$ NMR measurements were carried out in deuterated water at $25^{\circ} \mathrm{C}$ using a Bruker Advance $300 \mathrm{MHz}$ (7.05 T) spectrometer at the operating frequencies of 300.13 and $96.29 \mathrm{MHz}$, respectively. ${ }^{11} \mathrm{~B}$ NMR chemical shifts were referenced with respect to an external $\mathrm{BF}_{3}-\mathrm{OEt}_{2}$ standard at $0.0 \mathrm{ppm}$.

A standard BVT 3000 variable temperature control unit with an accuracy of $\pm 0.5^{\circ} \mathrm{C}$ was used. Self-diffusion coefficients were determined using a Bruker DIFF30 probe supplied by a Bruker Great $1 / 40$ amplifier that can generate field gradients up to $1.2 \mathrm{~T} \mathrm{~m}^{-1}$. The pulse-gradient stimulated echo (PGSTE) sequence was used $[26,27]$. Self-diffusion coefficients were obtained by varying the gradient strength $(g)$ while keeping the gradient pulse length $(\delta)$ and the gradient pulse intervals constant within each experimental run. The data were fitted according to the Stejskal-Tanner Eq. (2):

$I / I_{0}=\exp \left(-D q^{2} t\right)$

where $I$ and $I_{0}$ are the signals intensities respectively in the presence and absence of the applied field gradient, $q=\gamma g \delta$ is the so-called scattering vector $(\gamma$ being the gyromagnetic ratio of the observed nucleus), $t=(\Delta-\delta / 3)$ is the diffusion time, $\Delta$ is the delay time between the encoding and decoding gradients, and $D$ is the selfdiffusion coefficient to be extracted. Errors on the self-diffusion coefficients were estimated around $2 \%$ on the basis of repeated measurements. Areas of the ${ }^{11} \mathrm{~B}$ NMR resonances, assuming they were Lorentzian in shape, were calculated using the programme Microcal $^{\mathrm{TM}}$ Origin $^{\mathrm{TM}}$ from Microcal Software (Northampton, MA). Typically, errors on areas were estimated around 5\%.

\subsection{SAXS experiments}

SAXS measurements were carried out with a HECUS S3-MICRO camera (Kratky-type) equipped with a position-sensitive detector (OED $50 \mathrm{M}$ ) containing 1024 channels of width $54 \mathrm{~mm}$. Cu $\mathrm{K} \alpha$ radiation $(\lambda=1.542 \AA)$ was provided by an ultrabrilliant point micro-focus X-ray source (GENIX-Fox 3D, Xenocs, Grenoble), operating at a maximum power of $50 \mathrm{~W}(50 \mathrm{kV}$ and $1 \mathrm{~mA})$. The sample-to-detector distance was $281 \mathrm{~mm}$. The space between the sample and the detector was kept under vacuum during the measurements to minimize scattering from the air. Scattering curves were obtained in the q-range between 0.01 and $0.54 \AA^{-1}$. Gel samples were placed into $1 \mathrm{~mm}$ demountable cells having Kapton films as windows. The temperature was maintained at $25 \pm 0.1^{\circ} \mathrm{C}$ by a Peltier controller. The effect of the beam onto the samples during the collection of SAXS curves was evaluated by collecting some spectra at different acquisition times and no differences were observed, indicating that the samples were not damaged by the beam. All SAXS curves were modelled according to a Lorentzian model (Eq. (3)).

$I(q)=\frac{I_{0}}{1+(q L)^{2}}+b k g$

where $I_{0}$ is the scattering intensity at $q=0, L$ is the correlation length which corresponds in a semi-diluted solution to the average distance between neighbouring entanglement points and bkg is a q-independent instrumental background term.[28-30]
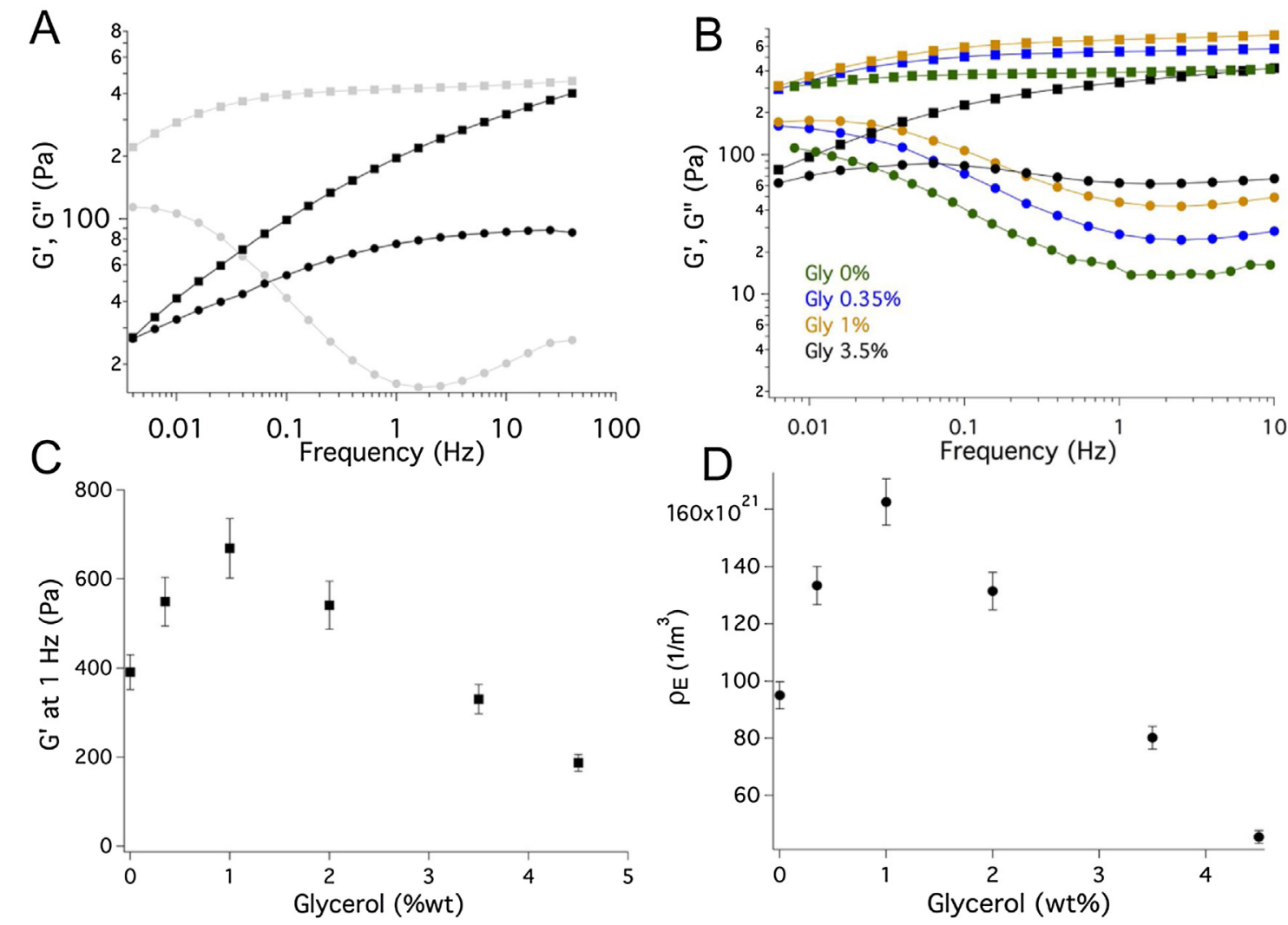

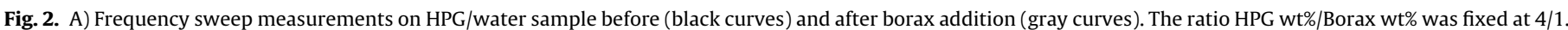

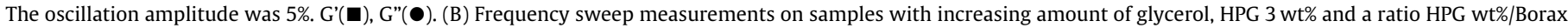

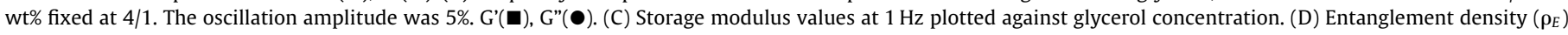
values plotted against glycerol concentration. 


\section{Results and discussion}

\subsection{Formulation and characterization}

The dynamic mechanical properties of HPG-based systems were investigated by means of frequency sweep oscillation tests. They provide information about the dependence of the storage modulus (G') and of the loss modulus (G") on the frequency of the applied shear perturbation. Fig. 2 A shows the trend of the storage modulus $G$ ' and the loss modulus $G$ " at a constant strain of $5 \%$ as a function of the applied shear perturbation. The polymer concentration in both samples was $3 \mathrm{wt} \%$ while the ratio HPG wt\%/Borax wt\% was fixed at $4 / 1$ as reported in literature for the formulation of similar crosslinked systems $[10,23]$.

The mechanical behaviour of HPG/water system was investigated before and after the addition of borax that acts as crosslinker. Borate anions induce the formation of covalent intermolecular junctions between the polymer chains through a di-diol condensation reaction. This results in a more rigid network and in a decrease of the viscous character of the samples: these covalent crosslinks, together with the intramolecular and intermolecular hydrogen bonding interactions that occur between the hydroxyl groups of the HPG chains, lead to an increase of the elastic behaviour and of systems stiffness. From the rheological point of view, the increase of borax determines the increase of the solid like behaviour of the system, with a huge increase in the Storage modulus G' as clearly indicated by the frequency sweep curves reported in Fig. S2.

To modify the system's stiffness, tuning the mechanical behaviour of the sample, glycerol was introduced as plasticizer, and its role in the viscoelastic properties of the formulation was systematically investigated by means of oscillating rheological test. Frequency sweep tests were carried out on systems containing different glycerol concentrations, while keeping HPG at $3 \mathrm{wt} \%$ and borax at $0.75 \mathrm{wt} \%$. The trend of the Storage and of the Loss moduli G' and G", at a constant strain of 5\% (in the linear viscoelastic range), as a function of the frequency is reported in Fig. $2 \mathbf{B}$, where the change of the elasticity of the systems induced by the addition of glycerol can be observed. In Fig. 2C, the Storage modulus ( $\left.G^{\prime}\right)$ at $1 \mathrm{~Hz}$ is plotted as a function of glycerol concentration. The presence of two different regimes is evident: at glycerol concentrations lower than $1 \mathrm{wt} \%$, a progressive increase of the stiffness of the system is observed indicating a structuring role of this additive. Above this threshold, the plasticizing effect of glycerol prevails as indicated by the decrease of G'. Fig. 2D shows the trend of the entanglement density $\rho_{E}$ as a function of the glycerol content. The entanglement density is equal to $k T G_{1 H z}^{\prime}$ where $\mathrm{k}$ is the Boltzman constant, $\mathrm{T}$ is the absolute temperature and $G_{1 H z}^{\prime}$ is the value of the elastic modulus $\mathrm{G}^{\prime}$ at $1 \mathrm{~Hz}$ [28]. The increase of $\rho_{E}$ at low glycerol content (up to $1 \mathrm{wt} \%$ ) indicates an increase of the complexity of the network confirming the structuring role of glycerol at low concentrations. A higher content of this plasticizer induces a progressive decrease of the entanglement density.

Systems containing different glycerol amounts were deeper investigated as a function of temperature between $25^{\circ} \mathrm{C}$ and $60^{\circ} \mathrm{C}$ by means of the Time Temperature Superposition (TTS) approach, to observe the temperature-dependence of viscoelastic properties of these fluids and, according to Eq. (1), to calculate the Activation Energy associated to the relaxation process. In Figs. S3-S7 the isothermal frequency sweep measurements and the master curves for samples containing HPG (3 wt\%)/borax (0.75 wt\%)/water and increasing amounts of glycerol are reported. The Activation Energy trend is reported in Fig. $3 \mathbf{A}$ : we observed that the Ea progressively decreases as glycerol concentration increases above $1 \mathrm{wt} \%$ confirming that above this threshold the driving factor is the plasticizing role of glycerol that cause a decrease of the Ea of the investigated systems.

With the aim of clarifying the role of glycerol in the crosslinking mechanism, an extensive ${ }^{11} \mathrm{~B}$ NMR study was carried out on samples containing increasing amount of glycerol. There are several papers in literature about the interaction between borax and hydroxyl groups [31 and refs therein] Several of them focus on crosslinked systems such as gel-like materials based on poly(vinyl alcohol) (PVA) [18,32] and on borax-galactomannan based gels [12].

As shown in Fig. $4 \mathbf{A}$, the ${ }^{11} \mathrm{~B}$ NMR spectrum of the HPG polymer/borax system in water is characterized by one intense resonance at $11.60 \mathrm{ppm}$ and a small resonance emerging at $5.55 \mathrm{ppm}$, being the downfield resonance due to the borate/boric acid molecular species, and the other to the cross-linked borate [33]. The addition of glycerol $(0.5 \mathrm{wt} \%)$ causes strong alterations of the NMR spectrum, namely a downfield shift of the free boron species resonance at $13.88 \mathrm{ppm}$, and the appearance of a new NMR signal at $9.77 \mathrm{ppm}$ that can be assigned to the formation of a glycerol-borate complex (see below). A dramatic increase of the cross-linked borate species with respect to the free borate/boric acid, as determined by the area of the NMR resonance signals at 13.88 and $5.55 \mathrm{ppm}$ (see Table S1), is also observed. With increasing glycerol concentration, we notice a progressive decrease of the amount of non-complexed boron species whose NMR signal undergoes a significant downfield shift, whereas no significant chemical shift changes are observed for the two complexed Borate species [33]. Conversely, the amount of glycerol-borate complex increases, whereas that of HPG-crosslinked increases up to $2 \mathrm{wt} \%$ of glycerol. For glycerol content higher than $2 \mathrm{wt} \%$ only the amount of glycerol-borate complex increases suggesting a very high stability of this new complex. Remarkably, the significant downfield shift of the non-complexed boron species indicates a change in the borate/boric acid ratio, with an increase in the uncharged species [33]. Such a phenomenon was

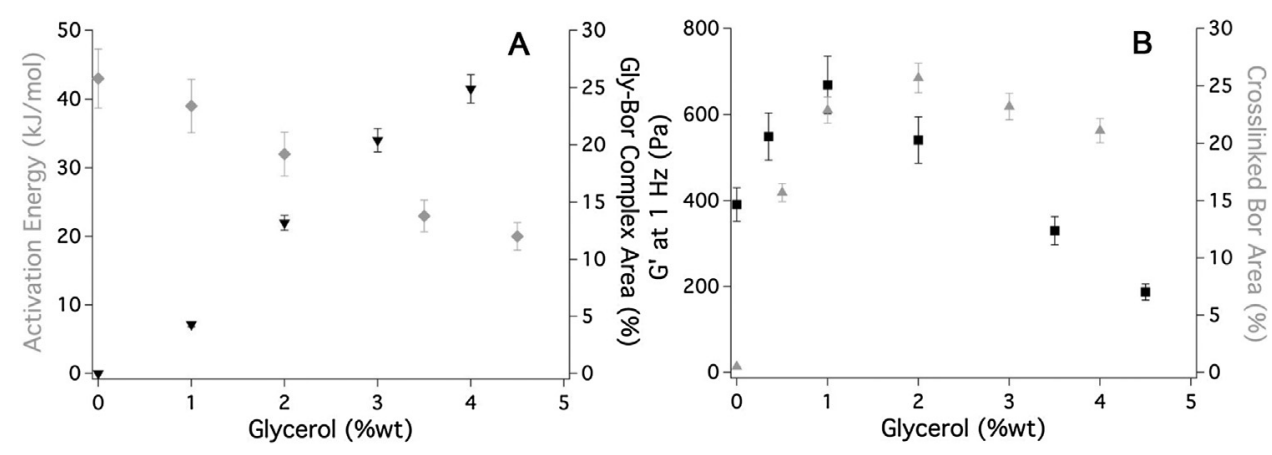

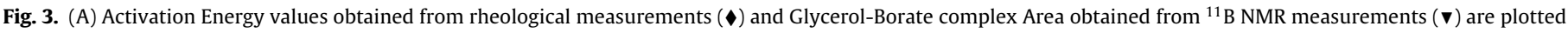

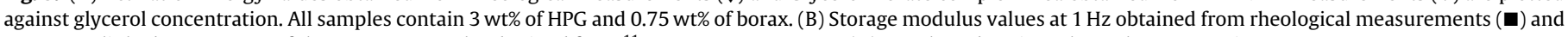
HPG-Crosslinked Borate Area of the resonance peaks obtained from ${ }^{11} \mathrm{~B}$ NMR measurements ( $\boldsymbol{\Lambda}$ ) are plotted against glycerol concentration. 

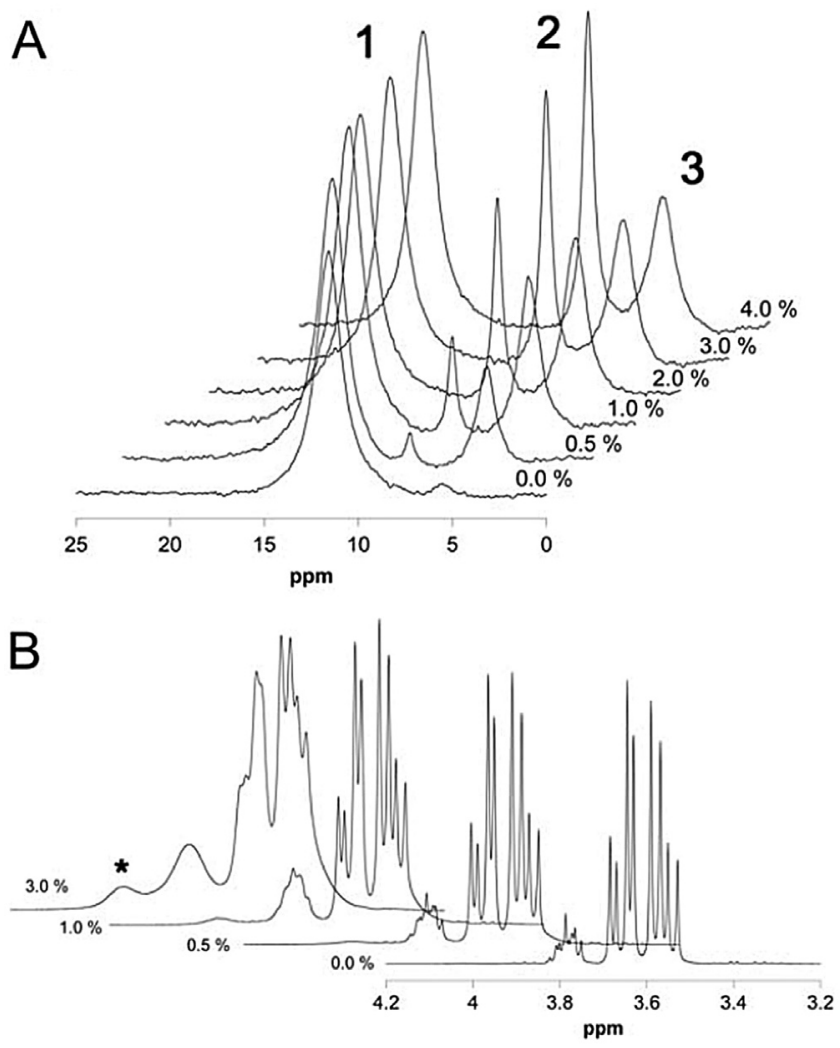

Fig. 4. (A) ${ }^{11} \mathrm{~B}$ NMR stacked plot spectra of the polymer/water system containing $3 \mathrm{wt} \%$ of HPG, $0.75 \mathrm{wt} \%$ of borax and increasing amounts of glycerol (indicated as wt\%). Peaks attribution: non-complexed boron (1), glycerol-borate complex (2), HPG-crosslinked borate (3). (B) ${ }^{1} \mathrm{H}$ NMR stacked plot spectra of the glycerol/water system containing increasing amounts of borax (wt\%). The star indicates a new resonance attributed to the glycerol-borate complex.

previously suggested to be related to the less attractive environment to borate ions originated by the addition of organic liquids $[18,34]$.

In other words, the addition of glycerol deeply affects the borate/boric acid equilibrium since the $\mathrm{B}(\mathrm{OH})_{4}^{-}$anion is preferentially involved in the formation of both the glycerol-borate complex and the HPG-crosslinked species. Clearly the addition of glycerol, even in small amount, may also alter the solvent properties such as, for instance, the dielectric constant. NMR data allow us to deeper understand the meaning of rheological data. From Fig. 3B we observe how the addition of glycerol up to $1 \%$ induced an increase of entanglement density resulting in an increase of system elastic behaviour, while a strong decrease of $G^{\prime}$ occurred at higher concentrations. In the same graph, we can see that the area of the crosslinked borate, measured by ${ }^{11} \mathrm{~B}$ NMR, follows the same trend, indicating that a fraction of borax is not anymore acting as a crosslinker in the polymeric network.

This behaviour may be ascribed to a possible double role of glycerol in the crosslinking mechanism. On one hand, at low concentrations, glycerol molecules seem to act as a structuring agent, inducing the crosslinking of borax and improving the elastic behaviour of the system. On the other hand, at concentration higher than $1 \mathrm{wt} \%$, glycerol seems to behave mainly as a plasticizer, reducing the density of the covalent borate bridges and then causing a decrease in the sample stiffness. The trend of $\tan \delta$ ( where $\tan \delta=G^{\prime \prime} / G^{\prime}$ ) at $1 \mathrm{~Hz}$, reported in Fig. S8, confirms a change in the viscoelastic behaviour for $\mathrm{HPG} / \mathrm{H}_{2} \mathrm{O}$ /borax based systems upon increasing the amount of glycerol. In particular, above $1 \%$, an increase of G" modulus is observed, resulting in less rigid samples.
Moreover, the glycerol-borate complex area increases with glycerol(Fig. 3A), confirming that borax is sequestered from the glycerol to create a complex. This leads to a decrease of the Activation Energy in the relaxation processes (Fig. 3A). When the complex formation begins to be much favoured, by increasing the amount of available glycerol, we see a sharp decline in the modulus G' in rheological measurements, and a slight decrease of crosslinked boron fraction in ${ }^{11} \mathrm{~B}$ NMR measurements (Fig. 3B). The two phenomena, which contribute to the variation of G', seem to proceed in the same direction up to a glycerol concentration between $1 \%$ and $2 \%$, then the formation of the glycerol-borate complex becomes favoured over the borax crosslinking mechanism.

To confirm the glycerol-borate complex formation, glycerol aqueous solutions were investigated before and after borax addition measuring the self-diffusion coefficient $D$ of glycerol by ${ }^{1} \mathrm{H}$ PGSTE NMR. In the analysed samples glycerol was maintained at a fixed concentration in deuterated water, while the amount of borax was increased. As reported in Table S2 the strong interaction between glycerol and borate induces an evident decrease in the glycerol motions that can be already observed at low glycerol-toborate molecular ratio, and becomes more significant as the borate concentration increases. Interestingly, upon the addition of borax a new downfield resonance started to grow (see Fig. 4B). It is worth noticing that borax in (deuterated) water does not show any ${ }^{1} \mathrm{H}$ NMR signal. Therefore, the new signal was attributed to the glycerol borate complex, and implicitly accounts for its high stability, being the fingerprint of a stable complex rather than a loose assembly in fast exchange between the bound and free form. In addition, the formation of the glycerol borate complex is confirmed by the progressive broadening of the width at half high and the consequent coalescence of the NMR signals observed in the ${ }^{1} \mathrm{H}$ NMR spectra as the borax concentration is increased (Fig. 4B). Indeed, such a loss of resolution is related to the more rapid spin-spin relaxation rates, in turn, caused by a) the reduced mobility experienced by the glycerol molecules because of the formation of the complex with borate ions, and particularly by b) the effect of the quadrupolar relaxation of the boron nucleus on the nearby nuclei.

After the dynamical characterization of systems containing increasing amount of glycerol, we focused on the role of the polymer in our formulations. To understand the effect of HPG concentration on the mechanical properties of the HPG based systems, frequency sweep measurements were carried out on a series of samples prepared with different amount of HPG (Fig. 5), while keeping the HPG/borax ratio at $4 / 1$ and glycerol at $3.5 \mathrm{wt} \%$. This value was selected in view of a potential application [18].

Fig. 5A indicates that the increase in HPG concentration doesn't significantly alter the shape of the shear moduli although its value increases upon increasing the HPG concentration, as also indicated by the decrease in the $\tan \delta$ values (see Fig. S9). Moreover, for HPG concentrations lower that $3 \mathrm{wt} \%$, two different regimes can be observed (Fig. 5A): at $\omega>\omega_{c}$ (where $\omega_{c}$ is the crossover frequency between the $G^{\prime}$ and $G$ " curves), G'>G', meaning that the elastic behaviour prevails; at $\omega<\omega_{c}$ where $G^{\prime}<G^{\prime \prime}$, the viscous character is predominant. Fig. 5A also shows that upon increasing the HPG concentration the crossover between G' and G" curves shifts towards lower frequency values, indicating an increase of the mean relaxation time that is equal to $1 / \omega_{c}$. In particular, the mean relaxation time moves from $1.6 \mathrm{~s}$ for the sample HPG1\% to $28.6 \mathrm{~s}$ for HPG $2 \%$. These values are usually related to sticky reptation dynamics [28]. Furthermore, for a HPG content higher than $3 \mathrm{wt} \%$, the crossover between G' and G" moves to not instrumentally accessible frequencies, indicating an increase of the solid-like character of the system. Moreover the increase of G' observed by increasing the HPG concentration indicates an increase in the complexity of the structure of the investigated systems and an increase in the strength of the $3 \mathrm{D}$ network due to the increase of the spatial density of the borate- 

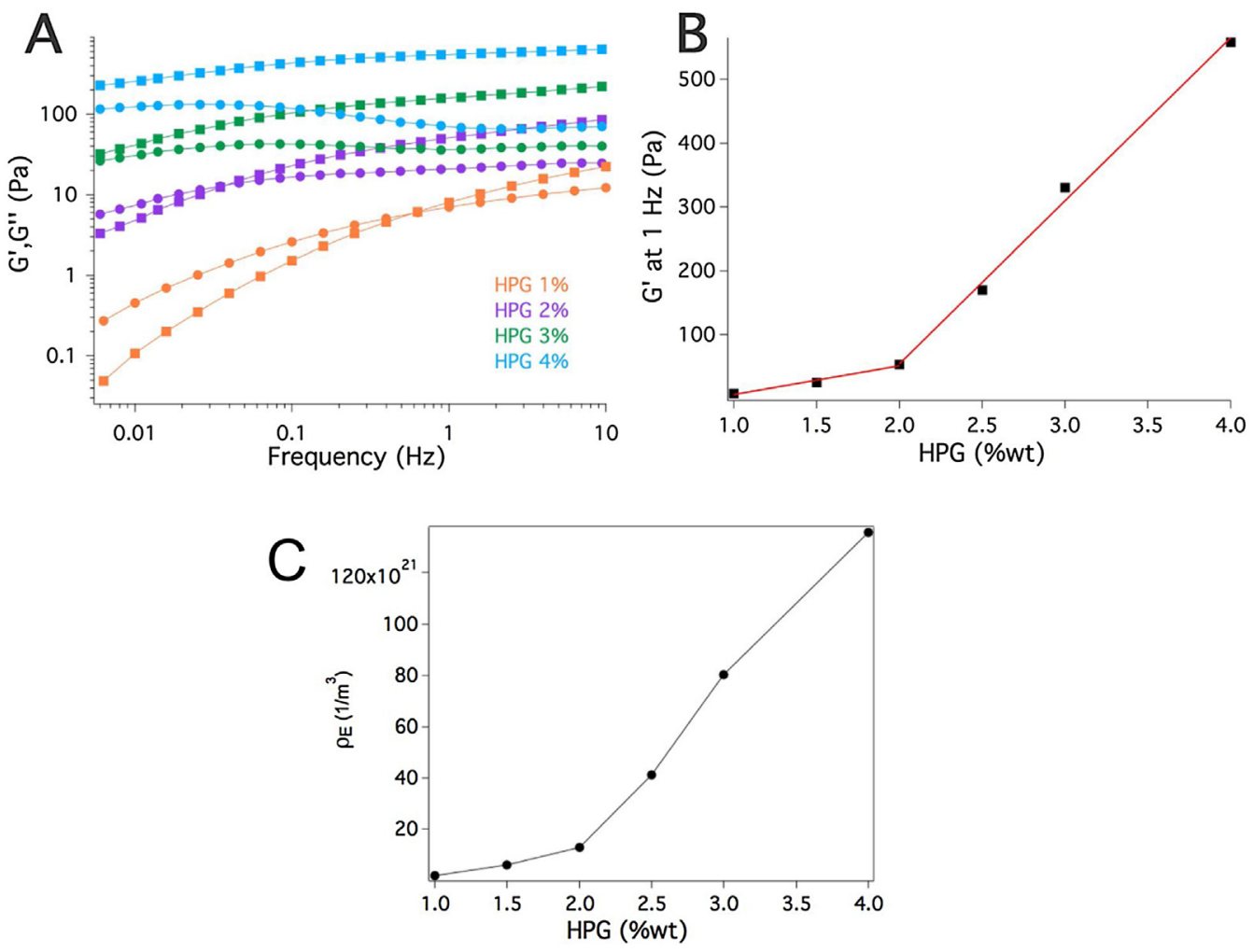

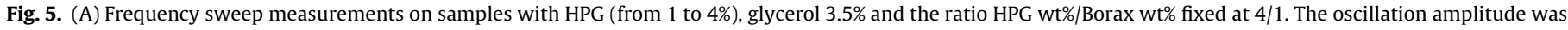
$5 \%$. G'(घ), G'( ). (B) Storage modulus values at $1 \mathrm{~Hz}$ as a function of HPG concentration. (C) Entanglement density ( $\rho_{E}$ ) values as a function of HPG concentration.

modulated cross-linking $\left(\rho_{e}\right)$ between the HPG chains [35]. Fig. 5B reports the trend of the elastic modulus $\mathrm{G}^{\prime}$ at $1 \mathrm{~Hz}$ as a function of the HPG concentration; the graph shows that $\mathrm{G}^{\prime}(1 \mathrm{~Hz})$ is nearly constant up to $2 \mathrm{wt} \%$ of HPG, while above this threshold (indicated as $\left.C^{*}\right)$, the drastic increase in $\mathrm{G}^{\prime}(1 \mathrm{~Hz})$, that reaches a value of $600 \mathrm{~Pa}$ at $4 \mathrm{wt} \% \mathrm{HPG}$, indicates the formation of an extended 3D polymer network. This is also confirmed by the trend of the entanglement density $\rho_{E}$ value that strongly increases for a HPG content higher than 2 wt\% (Fig. 5 C).

There is a considerable literature on the dependence of the $\mathrm{G}^{\prime}$ modulus on polymer concentration in solution [35 and refs therein]. In most cases, the dependence of $G^{\prime}$ is described by a power law: $G^{\prime} \propto \phi^{\mathrm{n}}(4)$ where $\phi$; is the polymer concentration expressed as wt\% [36]. For rigid networks having a fibrillary morphology the exponent $\mathrm{n}$ is strictly related to the fractal dimension $\mathrm{D}_{\mathrm{F}}$ of the object connecting at the junction (5) [37-39].

$G^{\prime} \propto \phi^{n}$

$G^{\prime} \propto \phi^{\left(3+D_{F}\right) /\left(3-D_{F}\right)}$

In Fig. S10 the trend of the G' values at $1 \mathrm{~Hz}$ are plotted on a $\log / \log$ scale as a function of the polymer concentration. From the obtained slope $(n)$, a fractal dimension of 1.6 is calculated, and this suggests, accordingly to Jones and Marques theory [38], a network with objects connected by rigid junctions, for which an enthalpic elasticity occurs [37].

The rheological properties of a selected system containing HPG $3 \%$ /borax $0.75 \%$ /glycerol $3.5 \%$ were also investigated as a function of temperature between $25^{\circ} \mathrm{C}$ and $60^{\circ} \mathrm{C}$, to deepen the temperaturedependence of viscoelastic properties of these fluids and to obtain the activation energy (Ea) associated to the relaxation process through the TTS approach (Eq. (1)). The frequency sweep curves and the master curves for samples investigated are reported in Figs.
S3, S6, S11 and S12, while the activation energy values for samples with and without glycerol and borax are listed in Table S3.

The addition of borax to the HPG dispersion in water (sample 2 and sample 1, respectively) lead to an increase of the Ea value, confirming the ability of borax in increasing the systems' stiffness (see Table S3). The addition of glycerol to sample 2 determined a significant decrease of the Ea value (sample 3). It is worth noting that both the formulations containing glycerol (samples 3 and 4), are characterized by activation energy values that are much lower than those of the corresponding systems that don't contain glycerol (system 2 and 1, respectively). As a matter of fact, over $2 \mathrm{wt} \%$, glycerol creates a stable complex with borate, resulting in less rigid systems, as shown by NMR measurements. NMR results are also confirmed by the comparison between Ea values of samples 3 and 4: the addition of borax to a system containing glycerol does not increase significantly the activation energy of the relaxation process, differently from what happened for samples 2 and 1 . This is due to the fact that borate is partially sequestered by glycerol and it is not entirely involved in the crosslinking of HPG.

SAXS curves have been collected on HPG aqueous binary solutions before and after the addition of the plasticizer and the crosslinker to better understand the role of the components on the structure of the studied systems. In Fig. 6, SAXS profiles and the best fitting curves according to Eq. (3) are reported. Fitting results are reported in Table 1 . As reported in literature [30], in a semi-diluted polymeric solution, the mesh size (indicated by the Screening Length $(\mathrm{L})$ ) corresponds to the average distance between neighbouring crosslinking points of the 3D polymeric network.

For what concern samples 1 and 2, the addition of borax does not significantly change the mesh size of the HPG aqueous dispersion, even if the entanglement density increases of almost two order of magnitude. This indicates that in the absence of glycerol, the interactions occurring between the HPG chains are the driving factor in determining the mesh size of the network, while the value of $\rho_{E}$ 

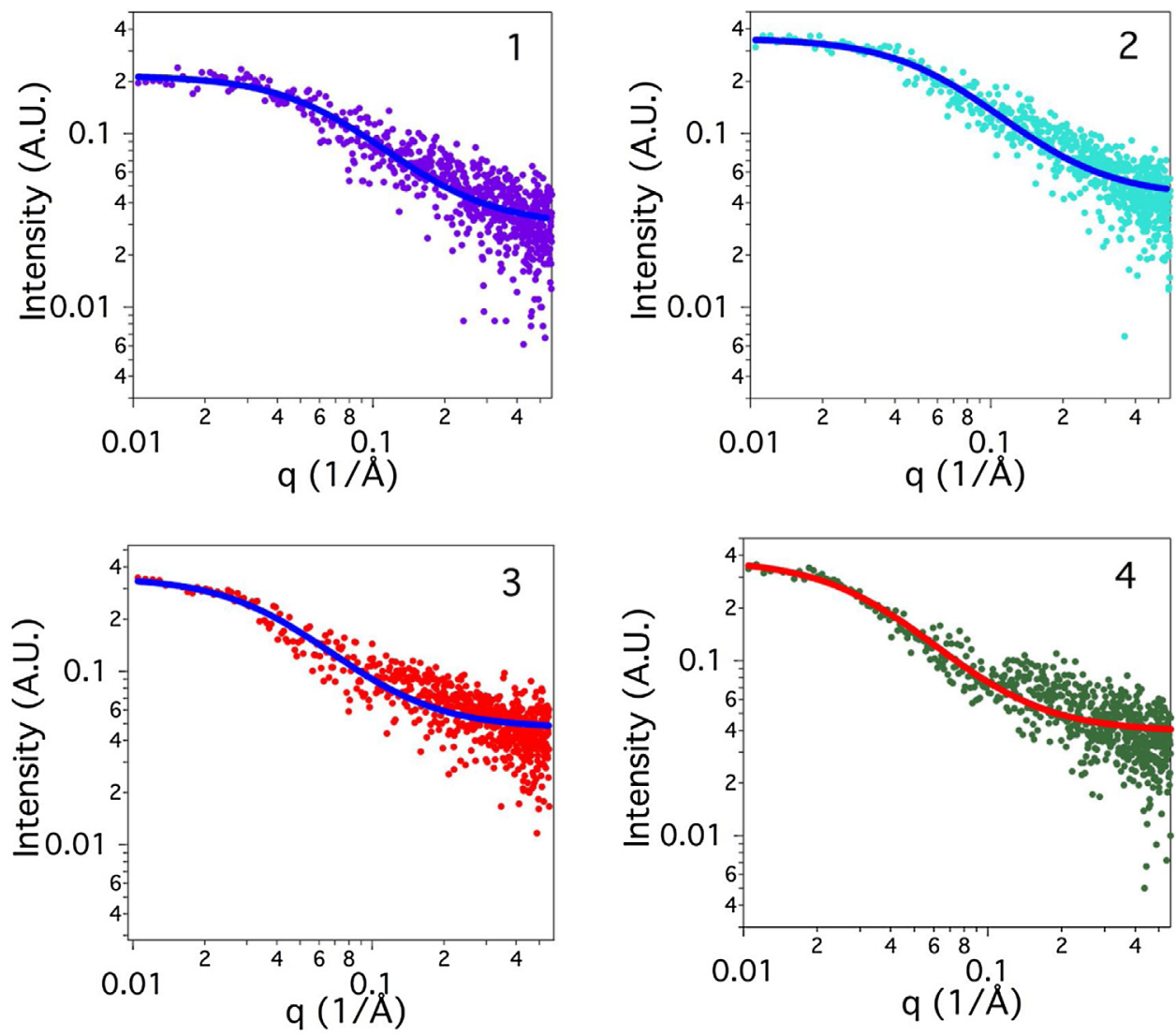

Fig. 6. Comparison between SAXS curves samples containing HPG/water (1), HPG/borax/water (2), HPG/glycerol/borax/water (3) and HPG/glycerol/water (4).

Table 1

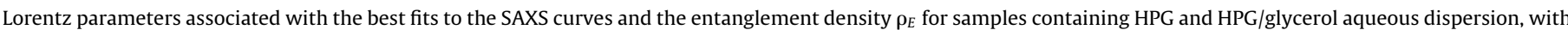
and without borax as crosslinker.

\begin{tabular}{|c|c|c|c|c|c|}
\hline SAMPLE & COMPOSITION & Scale factor I0 & Screening Length $\mathrm{L}(\AA)$ & $\mathrm{Bkg}$ & ${ }_{\rho E}\left(1 / m^{3}\right)$ \\
\hline 1 & HPG 3 wt\% in water & $0.19 \pm 0.03$ & $14.5 \pm 0.4$ & 0.03 & $4.1 \times 10^{20}$ \\
\hline 2 & HPG 3 wt\%/borax/water & $0.31 \pm 0.04$ & $15.2 \pm 0.3$ & 0.04 & $95 \times 10^{21}$ \\
\hline 3 & HPG 3 wt\%/glycerol 3.5 wt\%/borax/water & $0.30 \pm 0.06$ & $24.5 \pm 0.6$ & 0.05 & $80 \times 10^{21}$ \\
\hline 4 & HPG 3 wt\%/glycerol $3.5 \mathrm{wt} \%$ in water & $0.34 \pm 0.07$ & $28.9 \pm 0.7$ & 0.04 & $1.6 \times 10^{20}$ \\
\hline
\end{tabular}

mainly depends upon the presence of the crosslinking agent. On the contrary, in the presence of glycerol (sample 3 and 4), borax leads to a significant decrease in the screening length of the polymeric network. Moreover, the addition of glycerol leads to a huge increase of the mesh size (compare sample 1 with sample 4 and sample 2 with sample 3), confirming the ability of this additive to interpose among HPG chains, reducing the entity of the interactions between the HPG monomers (mainly due to $\mathrm{H}$ bonds), therefore acting as a plasticizer.

\subsection{Application test}

In the last decades, several advanced and innovative systems for the conservation of cultural heritage have been proposed [40-42]. In particular hydro and organogels have been proposed as a feasible alternative to overcome the limits of traditional methods for the selective removal of patinas from surfaces of artistic and historical interest [43-47].

The characterization of HPG/glycerol/borax gel-like systems shows that their properties can be tuned, even dramatically, by varying the concentration of the components. From the applicative point of view this is a very attractive feature of these systems. Therefore, a preliminary applicative test using a gel-like formula- tion with HPG (3 wt\%), borax (0.75 wt\%), and glycerol (3.5 wt\%) was carried out. This system was chosen because it can be stretched without breaking (see Fig. S13), which is an appealing feature for the application on curved surfaces. Moreover, the value of the intrinsic elastic modulus of this system allows its peeling from the cleaned surface in one step [18]. It is worth noting that this formulation has a pH between 8 and 9 , which is completely compatible with most of objects of historical and artistic interest.

After the fire that almost destroyed La Fenice theatre (Venice) in 1996, artworks and decorations that belonged to the beautiful historical building were altered by a dark coating, resulted from dust and particulate matter generated from the fire. The stucco of La Fenice theatre is a rough, porous material, as can be seen from Fig. 7A. The first attempt to remove the surface patina was carried out by following a traditional procedure based on the use of a cotton swab soaked with demineralized water. In that way, it was possible to obtain the softening of the dirt layer, which was then absorbed by the porous matrix, resulting in an unsatisfactory cleaning of the decoration (Fig. 7B). A second cleaning test was then carried out by means of a gel-like HPG-system that, according to the Washburn equation, due to its high viscosity, allowed the minimization of the penetration of the water into the porous support. The HPG/glycerol/borax system was applied on the stucco surface 

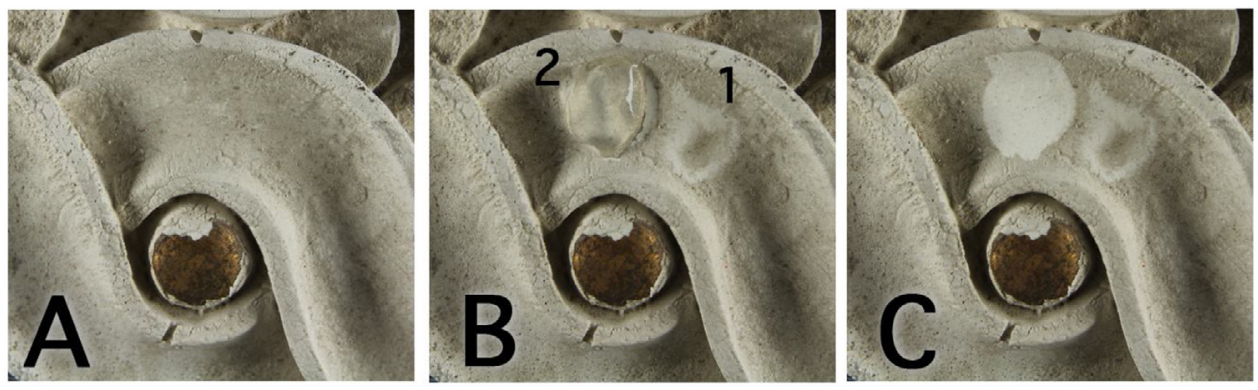

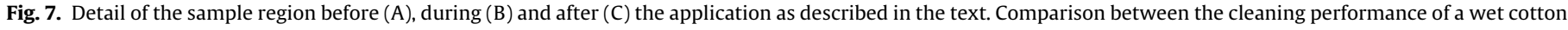
swab (1) and of the HPG based system (2).

with a spatula and left on the surface for about $1 \mathrm{~min}$ (see Fig. 7B); after the application, the system was removed in one step through a simple peeling action. The comparison between the cleaning action of our system and that of neat water applied by means of a wet cotton swab can be observed in Fig. 7C. The promising results of this preliminary test suggest a possible application of this formulation for the selective cleaning of the surface making these gel-like systems particularly interesting in the field of restoration.

\section{Conclusions}

A rheological, dynamic, and structural characterization of water based gel-like systems containing hydroxypropyl guar gum (HPG), borax, and glycerol is reported. From rheological measurements, we observed that the addition of borax to aqueous HPG dispersions lead to systems with increased stiffness, being borax a well-known efficient crosslinker for hydroxyl-rich polymers. With the aim of tuning the mechanical properties of HPG/borax gellike systems, variable amounts of glycerol were added and the overall effect on the systems was studied. Surprisingly, at low concentration (below 1\%), glycerol acts a structuring agent, increasing the storage modulus. Above $1 \%$, it behaves as a plasticizer, as it might be expected. We then decided to deepen the role of glycerol in network formation and its interactions with borax, using ${ }^{11} \mathrm{~B}$ NMR and ${ }^{1} \mathrm{H}$ NMR PGSTE. In particular, from ${ }^{11} \mathrm{~B}$ NMR, the formation of a glycerol-borate complex was observed. When glycerol is higher than $1 \%$, the complex formation act as a driving force and occurs at the expenses of the crosslinking between borax and polymer chains, resulting in the decrease of the Storage modulus, as observed from rheological measurements. The formation of a stable glycerol-borate complex was confirmed by ${ }^{1} \mathrm{H}$ PGSTE NMR measurements, from which we calculated the diffusion of glycerol in borax aqueous solutions. The activation energies of the relaxation process obtained by Time Temperature Superposition (TTS) allow us to calculate the fractal dimension of the investigated systems that resulted to be the one typical of a network with objects connected by rigid junctions. A structural characterization carried out by SAXS experiments provided insight about the mesh size, which is influenced by both the presence of borax and glycerol. A preliminary applicative test for the removal of a dark patina from a stucco decoration belonging to La Fenice theatre (Venice) was then performed. The promising results obtained in these preliminary experiments suggest a possible future application of these formulations in the field of restoration.

\section{Conflict of interest}

None.

\section{Funding}

This work was supported by Center for colloid and surface science and by the European Union's Horizon 2020 research and innovation programme under grant agreement No 646063 [Nanorestart Project]. Sergio Murgia and Maura Monduzzi thank Fondazione Banco di Sardegna and Regione Autonoma della Sardegna (Progetti Biennali di Ateneo Annualità 2016, Fondazione Sardegna CUP F72F16003070002 and F72F16003060002). All authors greatefully thank the friend Prof. Piero Baglioni for its friendship and its constant fundamental contribution to the field of Soft Matter and the nanotechnologies applied to the Cultural Heritage Conservation.

\section{Appendix A. Supplementary data}

Supplementary data associated with this article can be found, in the online version, at https://doi.org/10.1016/j.colsurfb.2018.02. 025 .

\section{References}

[1] R.K. Proud'homme, V. Costien, S. Knoll, The effect of shear hystory on the rheology of hydroxypropyl guar gels, Polym. Aqueous Media Perform. Assoc. (1989) 89-112.

[2] J.E. Fox, Seed gums, thick, Gelling Agents Food 26 (1997) 262-283.

[3] C.H. Kucera, D.N. DeMott, Drilling fluid containing crosslinked polysaccharide derivative, US patent 4257903, 1981.

[4] T. Coviello, F. Alhaique, A. Dorigo, P. Matricardi, M. Grassi, Two galactomannans and scleroglucan as matrices for drug delivery: preparation and release studies, Eur. J. Pharm. Biopharm. 66 (2007) 200-209.

[5] H. Kono, F. Otaka, M. Ozaki, Preparation and characterization of guar gum hydrogels as carrier materials for controlled protein drug delivery, Carbohydr. Polym. 111 (2014) 830-840.

[6] T. Coviello, P. Matricardi, C. Marianecci, F. Alhaique, Polysaccharide hydrogels for modified release formulations, J. Control. Release 119 (2007) 5-24.

[7] G. Milcovich, F.E. Antunes, R. Farra, M. Grassi, F. Asaro, Modulating carbohydrate-based hydrogels as viscoelastic lubricant substitute for articular cartilages, Int. J. Biol. Macromol. 102 (2017) 796-804.

[8] Y. Cheng, K.M. Brown, R.K. Prud'homme, Characterization and intermolecular interactions of hydroxypropyl guar solutions, Biomacromolecules 3 (2002) 456-461.

[9] D. Risica, A. Barbetta, L. Vischetti, C. Cametti, M. Dentini, Rheological properties of guar and its methyl, hydroxypropyl and hydroxypropyl-methyl derivatives in semidilute and concentrated aqueous solutions, Polymer (Guildf) 51 (2010) 1972-1982.

[10] S. Wang, H. Tang, J. Guo, K. Wang, Effect of pH on the rheological properties of borate crosslinked hydroxypropyl guar gum hydrogel and hydroxypropyl guar gum, Carbohydr. Polym. 147 (2016) 455-463.

[11] R. Dietrich, M. Flury, Agent for removing solid particles, US patent 7541325 , 2009.

[12] E. Pezron, A. Ricard, F. Lafuma, R. Audebert, Reversible gel formation induced by ion complexation. 1. Borax-galactomannan interactions, Macromolecules 21 (1988) 1121-1125.

[13] T. Coviello, P. Matricardi, F. Alhaique, R. Farra, G. Tesei, S. Fiorentino, et al., Guar gum/borax hydrogel: rheological, low field NMR and release characterizations, E-XPRESS Polym. Lett. 7 (2013) 733-746.

[14] M. Shibayama, H. Yoshizawa, H. Kurokawa, H. Fujiwara, S. Nomura, Sol-gel transition of poly(vinyl alcohol)-borate complex, Polymer 28 (1988) 2066-2071. 
[15] G. Bocchinfuso, A. Palleschi, C. Mazzuca, T. Coviello, F. Alhaique, G. Marletta, Theoretical and experimental study on a self-assemblimg polysaccharideforming nanochannels: static and dynamic effect induced by a soft confinement, J. Phys. Chem. B 112 (2008)

[16] A. Palleschi, T. Coviello, G. Bocchinfuso, F. Alhaique, Investigation on a new scleroglucan/borax hydrogel: structure and drug release, Int. J. Pharm. 322 (2006) 13-21.

[17] G. Bocchinfuso, C. Mazzuca, C. Sandolo, S. Margheritelli, F. Alhaique, T. Coviello, et al., Guar gum and scleroglucan interactions with borax: experimental and theoretical studies of an unexpected similarity, J. Phys. Chem. B 114 (2010) 13059-13068.

[18] L. Angelova, B. Berrie, K. De Ghetaldi, A. Kerr, R.G. Weiss, Partially hydrolised poly(vinyl acetate)-boraxbased gel-like materials for conservation of art: characterization and applications, Stud. Conserv. 60 (2015) 227-244.

[19] E. Pezron, A. Ricard, L. Leibler, Rheology of galactomannan-borax gels, J. Polym. Sci. Part B Polym. Phys. 28 (1990) 2445-2461.

[20] V. Epure, M. Griffon, E. Pollet, L. Avérous, Structure and properties of glycerol-plasticized chitosan obtained by mechanical kneading, Carbohydr. Polym. 83 (2011) 947-952.

[21] C. Gao, E. Pollet, L. Av, Food Hydrocolloids Properties of glycerol-plasticized alginate fi lms obtained by thermo- mechanical mixing, Food Hydrocoll. 63 (2017) 414-420.

[22] M. Kapoor, D. Khandal, R. Gupta, P. Arora, G. Seshadri, S. Aggarwal, et al. Certain rheological aspects of functionalized guar gum, Int. J. Carbohydr. Chem. 2013 (2013) 1-15.

[23] S. Kesavan, R.K. Proud'homme, Rheology of guar and HPG cross-linked by borate, Macromolecules 2 (1992) 2026-2032.

[24] M. Rietjens, P.A. Steenbergen, Crosslinking mechanism of boric acid with diols revisited, Eur. J. Inorg. Chem. 116 (2005) 1162-1174.

[25] J. Dealy, D. Plazek, Time temperature superposition - a user guide, Rheol. Bull. 78 (2009) 16-31.

[26] S. Murgia, M. Monduzzi, F. Lopez, G. Palazzo, Mesoscopic structure in mixtures of water and 1-butyl-3-methyl imidazolium tetrafluoborate: a multinuclear NMR study, J. Solution Chem. 42 (2013) 1111-1122.

[27] S. Murgia, G. Palazzo, M. Mamusa, S. Lampis, M. Monduzzi, Aerosol-OT in water forms fully-branched cylindrical direct micelles in the presence of the ionic liquid 1-butyl-3-methylimidazolium bromide, Phys. Chem. Chem. Phys. 13 (2011) 9238-9245.

[28] E. Carretti, C. Matarrese, E. Fratini, P. Baglioni, L. Dei, Physicochemical characterization of partially hydrolyzed poly(vinyl acetate)-borate aqueous dispersions, Soft Matter 10 (2014) 4443-4450.

[29] M. Shibayama, Structure-mechanical property relationship of tough hydrogels, Soft Matter 8 (2012) 8030-8038.

[30] P.G. De Gennes, Scaling Concepts in Polymer Physics, Cornell University Press, Ithaca, New York, 1979 .

[31] P.J.P. Sci, Complex Formation in Polymer-Ion Solutions. 1. Polymer Concentration Effects, (1989) 1169-1174.
[32] S.G. Chaudri, B.H. Rajai, P.S. Singh, Nanoscale homogeneity of silica-poly(vinyl alcohol) membranes by controlled cross-linking via sol-gel reaction in acidified and hydrated ethanol, RSC Adv. 5 (2017) 65862-65869.

[33] M. Bishop, N. Shahid, J. Yang, A.R. Barron, Determination of the mode and efficacy of the cross-linking of guar by borate using MAS 11 B NMR of borate cross-linked guar in combination with solution 11 B NMR of model systems, Dalton Trans. (2004) 2621-2634.

[34] I. Natali, E. Carretti, L. Angelova, P. Baglioni, R.G. Weiss, L. Dei, Structural and mechanical properties of peelable organoaqueous dispersions with partially hydrolyzed poly(vinyl acetate)-borate networks: applications to cleaning painted surfaces, Langmuir 27 (2011) 13226-13235.

[35] B.A. Schubert, E.W. Kaler, N.J. Wagner, The microstructure and rheology of mixed cationic/anionic wormlike micelles, Langmuir 19 (2003) 4079-4089.

[36] P.G. de Gennes, P. Pincus, Scaling theory of polymer adsorption, J. Phys. 37 (1976) 1445-1452.

[37] J.-M. Guenet, Structure versus rheological properties in fibrillar thermoreversible gels from polymers and biopolymers, J. Rheol. (N. Y. N. Y.) 44 (2000) 947-960.

[38] J.L. Jones, C.M. Marques, Rigid polymer network models, J. Phys. France 51 (1990) 1113-1127.

[39] M. Pääkkö, M. Ankefors, H. Kosonen, A. Nÿkanen, S. Ahola, M. Östeberg, et al., Enzymatic hydrolysis combined with mechanical shearing and high-pressure homogenization for nanoscale cellulose fibrils and strong gels, Biomacromolecules 8 (2007) 1934-1941.

[40] T. Duncan, B. Berrie, R.G. Weiss, Soft, peelable organogels from partially hydrolyzed poly(vinyl acetate) and benzene-1,4-diboronic acid: applications to clean works of art, ACS Appl. Mater Interfaces 9 (2017) 28069-28078.

[41] P. Baglioni, D. Chelazzi (Eds.), Nanoscience for the Conservation of Works of Art, The Royal Society of Chemistry, London, UK, 2013.

[42] G. Poggi, N. Toccafondi, D. Chelazzi, P. Canton, R. Giorgi, P. Baglioni, Calcium hydroxide nanoparticles from solvothermal reaction for the deacidification of degraded waterlogged wood, J. Colloid Interface Sci. 473 (2016) 1-8.

[43] E. Carretti, L. Dei, Gels as cleaning agents in cultural heritage conservation, in: P. Terech, R.G. Weiss (Eds.), Molecular Gels: Materials with Self-Assembled Fibrillar Networks, Springer, New York, USA, 2005, pp. 929-938.

[44] L. Angelova, M. Leskes, B. Berrie, R.G. Weiss, Selective formation of organi, organo-aqueous, and hydro gel-like materials from partially hydrolysed poly(vinyl acetate)s based on different boron-containing crosslinker, Soft Matter 11 (2015) 5060-5066.

[45] P. Baglioni, E. Carretti, D. Chelazzi, Nanomaterials for art conservation, Nat. Nanotechnol. 10 (2015) 287-290.

[46] C. Mazzuca, G. Poggi, N. Bonelli, L. Micheli, P. Baglioni, A. Palleschi, Innovative chemical gels meet enzymes: a smart combination for cleaning paper artworks, J. Colloid Interface Sci. 502 (2017) 153-164.

[47] C. Berlangieri, E. Andrina, C. Matarrese, E. Carretti, R. Traversi, M. Severi, et al. Chelators confined into 80pvac-borax highly viscous dispersions for the removal of gypsum degradation layers, Pure Appl. Chem. 89 (2017) 97-109. 